\title{
Analysis of 14-bp HLA-G polymorphism in middle-eastern women with recurrent implantation failure
}

\author{
Rand Sulaiman $^{1}$, Tharaa Mohammad ${ }^{1}$, Marwan Alhalabi ${ }^{2 *}$
}

\begin{abstract}
${ }^{1}$ Kazan Federal University Institute of Fundamental Medicine and Biology, Kazan, Russian Federation, Russia ${ }^{2}$ Division of Reproductive Medicine, Embryology and Genetics, Faculty of Medicine, Damascus University, Damascus, Syria
\end{abstract}

Received: 13 September 2020

Revised: 01 November 2020

Accepted: 02 November 2020

\section{*Correspondence:}

Dr. Marwan Alhalabi,

E-mail: profalhalabi@icloud.com

Copyright: ( ) the author(s), publisher and licensee Medip Academy. This is an open-access article distributed under the terms of the Creative Commons Attribution Non-Commercial License, which permits unrestricted non-commercial use, distribution, and reproduction in any medium, provided the original work is properly cited.

\section{ABSTRACT}

Background: Implantation appears to be the major rate-limiting step in assisted reproductive techniques success. Recurrent implantation failure (RIF) is a common condition, being the failure to achieve a clinical pregnancy after transfer of four or more good-quality embryos in a minimum of two IVF cycles in a woman under the age of 40. Human leukocyte antigen G (HLA-G) is thought to play a key role in implantation by modulating cytokine secretion to enable the semi-allogeneic embryo to implant into the maternal endometrium. The aim of this study was to investigate the association between the 14-bp ins/del HLA-G polymorphism and rates of RIF. No similar studies were conducted nationally or regionally.

Methods: The study was designed as a case-control study. A proportion of 30 patients with RIF were selected as the experimental group. The control group included 15 patients with at least one pregnancy following IVF procedure. Peripheral blood samples were collected from both groups. DNA was extracted and the polymorphism analysis was performed using (AMPLI - HLA-G polymorphism) kit, then PCR products were electrophoresed on $2 \%$ agarose gel. statistical analysis was performed using SPSS statistics software.

Results: A total of 45 middle-eastern patients were recruited in this case-control study. High frequency of the polymorphism was found in both case $(76.6 \%)$ and control $(86.67 \%)$ groups, but with no significant difference between the two groups $(\mathrm{p}=0.429)$.

Conclusions: There is no correlation between the 14-bp ins/del HLA-G polymorphism and RIF, thus it may be considered as a normal polymorphism and might not affect the level of HLA-G.

Keywords: Assisted reproductive techniques, Recurrent implantation failure, Implantation, HLA-G polymorphism, middle east

\section{INTRODUCTION}

Since the birth of the first baby by IVF in 1978, this technique has seen a quantum leap in the last few decades by successfully treating infertility and increasing pregnancy rates. However, despite good quality embryos, unsuccessful implantations occur in more than half of infertile couples. ${ }^{1}$ The majority of IVF cycles lead to the transfer of embryos to the uterus, but most do not implant. Thus, the main cause of failure in IVF is implantation failure. ${ }^{2}$ The term recurrent implantation failure (RIF) has been used since 1983 to describe the failure of embryos to implant following $\mathrm{IVF}^{3}$

RIF is a form of refractory infertility with unknown aetiology, and it is defined as a failure to achieve a clinical pregnancy after transfer of four or more goodquality embryos in a minimum of two IVF cycles in a 
woman under the age of $40 .{ }^{4}$ The failure of implantation may be a consequence of embryo factors (genetics, sperm contribution, and immunologic factors) or uterine factors (uterine anatomy, endometrium, and thrombophilia), whereas the causes of RIF are still poorly known. ${ }^{5}$ It has been suggested that the abnormal maternal expression of genes associated with angiogenesis, immunity and apoptosis interferes with successful implantation. ${ }^{6}$

One of the central issues for a successful pregnancy is the prevention of maternal immune rejection, increasing evidence suggests that an unbalanced maternal immune response toward the embryo may cause its rejection. ${ }^{7}$ Recently, several lines of genetic-association studies have revealed association between the RIF risk and certain genetic polymorphisms, including tumor protein P53, cyclooxygenase-2 (COX-2), methylenetetrahydrofolate reductase (MTHFR), thymidylate synthase (TS), nuclear factor kappa B $(\mathrm{NF}-\kappa \mathrm{B})$, plasminogen activator inhibitor1, vascular endothelial growth factor, and leukocyte antigen-G. Among these candidate RIF susceptibility genes, the human leucocyte antigen-G (HLA-G) gene was one of the most extensively explored. ${ }^{8}$

Human leukocyte antigen-G (HLA-G) is a non-classical HLA class Ib molecule that is predominantly expressed in the fetal-maternal interface and plays an important role during implantation and maternal acceptance of the fetus. ${ }^{9}$ HLA-G includes four membrane-bound isoforms (HLAG1, G2, G3 and G4) and three soluble isoforms (HLA-G5, G6 and G7).10 The external part of the HLA$\mathrm{G}$ molecule consists of three parts, the $\alpha 1, \alpha 2$, and $\alpha 3$ domains (exons 2-4). The HLA-G full-length membrane protein is anchored in the cell membrane by the transmembrane region (exon 5). The cytoplasmic domain is encoded by exon 6 and the very first short part of exon 8. ${ }^{11}$ HLA-G is selectively expressed in tissues at the fetal-maternal interface, primarily in the extra-villous cytotrophoblast. ${ }^{12}$ HLA-G represents a key gene in embryo implantation, acting to modulate the local immune response, thereby suppressing an attack of the fetus by the maternal immune system. This suppression is accomplished by affecting maternal cytokine secretion to control the invasion by trophoblastic cells. ${ }^{13}$ The major HLA-G function is its ability to inhibit $T$ and $B$ lymphocytes, NK cells and antigen-presenting cells (APC). ${ }^{14}$

HLA-G is reported to possess immunosuppressive functions by an apoptotic effect on activated CD8+ T cells and suppression of an allo-proliferative response. ${ }^{11}$ The effect of HLA-G seems to be concentrationdependent. $^{7}$

HLA-G has a lower level of polymorphism than other HLA class molecules I. The extensively studied polymorphism in the non-coding region is a 14-bp insertion/deletion in 3'UTR of exon 8,11 which plays a role in HLA-G alternative splicing and affects HLA-G messenger RNA (mRNA) stability, consequently it gives rise to a smaller production of HLA-G.15 The polymorphism of HLA-G has been studied and the insertion of a 14-bp sequence within the $3^{\prime}$ untranslated region (UTR) region of HLA-G was reported to be involved with RIF, although the results are conflicting. ${ }^{16}$ A study conducted by Fan et al showed that there was statistically significant association between the HLA-G 14-bp polymorphism and RIF risk. ${ }^{8}$ In contrast, another study investigated the association between HLA-G gene polymorphism with repeated implantation failure (RIF), and the results indicated that HLA-G gene polymorphism does not clearly affect the risk for implantation failure in most couples who undergo ART. ${ }^{17}$ In this study, our objective is to investigate the association between 14-bp ins/del HLA-G polymorphism and RIF rates in the middle-east region, to better understand the contributing factors for RIF.

\section{METHODS}

\section{Subjects}

The study was conducted at assisted reproduction unit of Orient Hospital and Division of Reproductive Medicine, Embryology and Genetics of Faculty of Medicine of Damascus University, in the period between February 2018 to August 2018. The study was performed as a casecontrol experiment. We selected 30 women with RIF following IVF treatment as the experimental group. The control group included 15 women with at least one pregnancy following IVF procedure. The inclusion criteria were: patients under 40 years who underwent IVF treatment and ended in 2 RIF at least, patients with no thrombophilia or immunological diseases history and do not have any pathology that may affect implantation as endometriosis, fibroids, intra-uterine adhesion or abnormalities, and the cause for infertility was male factor for all patients. Subjects who were diagnosed with RIF due to anatomical, chromosomal, hormonal, infectious, autoimmune or thrombotic causes were excluded from the present study. The patients originate from Syria, Iraq, Palestine, Lebanon, Jordan, Saudi Arabia, United Arab Emirates and Qatar (Middle East). All participants gave informed consent, according to the protocol approved by local ethics committee at Damascus university and health ministry.

\section{Mutation analysis}

Peripheral blood samples were collected on EDTA tubes, and DNA was extracted using automated DNA extraction system (Sacace_SaMag-12). The 14b-p insertion/deletion HLA-G mutation analysis was performed using (AMPLI - HLA-G polymorphism) kit which contains the PCR reaction reagents, Taq Polymerase $(5 \mathrm{U} / \mu \mathrm{l})$ and three controls: ins/del HLA-G 14-bp, ins/ins HLA-G 14bp, and del/del HLA-G 14 bp. PCR was performed in $25 \mu \mathrm{l}$ reaction containing $23 \mu \mathrm{l}$ PCR mix and $2 \mu \mathrm{l}$ DNA sample. PCR reaction was performed in thermal cycler which is programmed for one cycle at $95 \mathrm{C}^{\circ}$ for $10 \mathrm{~min}$ then 35 
cycles of denaturation at $95 \mathrm{C}^{\circ}$ for $30 \mathrm{~s}, 60 \mathrm{C}^{\circ}$ for $30 \mathrm{~s}$ and $72 \mathrm{C}^{\circ}$ for $30 \mathrm{~s}$. Additional cycle at $72 \mathrm{C}^{\circ}$ for $10 \mathrm{~min}$ preceded the previous cycles. PCR products and the controls were electrophoresed on $2 \%$ agarose gel and after ethidium bromide staining PCR product bands were visualized under UV light.

\section{Statistical analysis}

Descriptive statistical analysis was performed using SPSS statistics software. Differences between the experimental and control groups were determined with the Independent sample t-test. To evaluate the relationship between either alleles or genotypes with the risk of having RIF, Chisquared test was performed for each genotype and allele against others. The value of $\mathrm{P}<0.05$ was defined as significant frequency difference between the two groups.

\section{RESULTS}

\section{HLA-G polymorphism}

Patients in control and study groups showed no difference in demographic data and controlled characteristics.

When comparing the mean age, FSH level, AMH level and the number of transferred embryos between the control and study groups, there were no statically significant differences between the two groups $(\mathrm{p}=0.064)$ (Table 1).

Table 1: Characteristics of patients in control and study groups.

\begin{tabular}{|llll|}
\hline Basal FSH level (ng/ml) & $\begin{array}{l}\text { PIF patients (study group) } \\
\mathbf{n}=\mathbf{3 3}(\text { mean } \pm \text { SD) }\end{array}$ & $\begin{array}{l}\text { Control group } \mathbf{n = 1 5} \\
(\text { mean } \pm \text { SD) }\end{array}$ & P value \\
\hline AMN level (ng/ml) & $7.0213( \pm 2.81021$ & $7.4167 \pm 2.70280$ & 0.841 \\
\hline Age (years) & $3.9300 \pm 3.75828$ & $3.9233 \pm 2.72949$ & 0.995 \\
\hline Number of transferred embryos & $33.8000 \pm 4.78792$ & $33.0833 \pm 5014266$ & 0.682 \\
\hline
\end{tabular}

Table 2: The frequency of the three types of HLA-G gene mutations (ins/del cins/ins ‘del/del) in study ( $R$ IF cases) and control groups.

\begin{tabular}{|llll|}
\hline Mutation type & RIF patients (study group) $\mathbf{n = 3 0}$ & Control group n= 15 & P value \\
\hline ins/del & $47.82 \%$ & $30.77 \%$ & 0.502 \\
\hline ins/ins & $26.08 \%$ & $53.84 \%$ & 0.063 \\
\hline del/del & $26.08 \%$ & $15.38 \%$ & 0.581 \\
\hline Total mutation frequency & $76.67 \%$ & $86.67 \%$ & 0.429 \\
\hline
\end{tabular}

The frequency of HLA-G mutation is equally distributed between control and study groups.

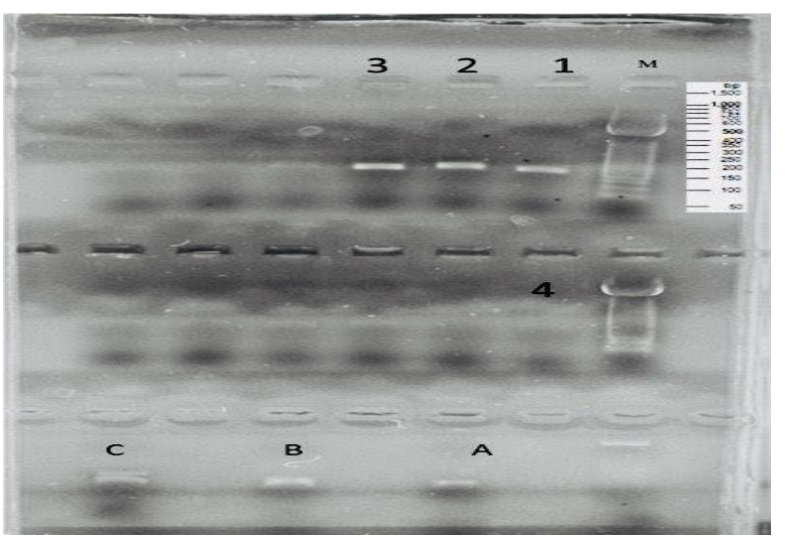

Figure 1: Electrophoresis results of certain samples and positive controls. (M) DNA ladder 50bp; 1, 2 and

3: positive samples (ins/ins) mutation; 4: negative sample; (A) positive control (del/del 196bp); (B) positive control (ins/ ins 210bp) 210bp; (C) positive control (ins/del 210 and 196 bp).
Polymerase chain reaction (PCR) with specific primers was used to detect the three types of HLA-G gene mutations in samples, the results of subsequent gel electrophoresis are shown in (Figure 1). There were no statically significant differences when comparing the frequency of the mutation between control and study groups $(\mathrm{p}=0.429)$ (Table 2).

\section{DISCUSSION}

RIF is considered a major risk threatening the success of ART. Many studies have been carried out on the investigation of the possible causes of RIF cases. Several immunological mechanisms have been investigated to elucidate the aetiology of a large proportion of RIF cases. The purpose of this study was to investigate the association between the 14-bp ins/del 3'UTR HLA-G polymorphisms and RIF cases in middle-eastern patients, which is still a matter of conflict whether it has an effect on the implantation process and what is the mechanism by which it may effect. 
Many studies have focused on the role of HLA-G molecules on implantation, and the association between HLA-G gene polymorphisms and soluble HLA-G protein levels in the maternal serum and follicular fluid with the cases of embryo implantation failure. ${ }^{13,14}$ The early embryo (two-day old) express the sHLA-G protein by translating the residual maternal mRNA. This indicates the importance of detecting the maternal over the paternal polymorphism, thus the polymorphism could be detected using maternal peripheral blood samples or follicular fluid samples. ${ }^{17}$ Rizzo et al results indicated a strong relation between sHLA-G concentration in the follicular fluid for every individual follicle and its concentration in the resulted embryo culture medium supernatant, which suggest the possibility of using the sHLA-G protein as a tool for oocyte selection in IVF. ${ }^{18}$ These results contradict with Ouji-Sageshima et al. study, which suggest no relation between SHLA-G concentration in the follicular fluid and the success of implantation. ${ }^{19}$ Comparing the frequency of the 14-bp polymorphism between the experimental and control groups in our study, no significant difference was found, which indicates no correlation between the polymorphism and the RIF cases. Thus, this polymorphism may be ineffective on the production of sHLA-G.

There are three types of the studied polymorphism, which are either homozygote genotypes (ins/ins, del/del) or heterozygote genotype (ins/del). The frequencies of these alleles were studied in both experimental and control group. Our results showed that ins/del allele is the most frequent allele among the experimental group, and ins/ins allele is most frequent among the control group but when comparing the two groups, no significant difference was found, which indicates no dominance of one allelic type among the RIF patients in comparison with the control group. This is in accordance to a study by Sipak-Szmigiel et al. that showed no association of the 14-bp ins/ins or ins/del genotype with RIF. ${ }^{20}$ Our result contradicted with Enghelabifar et al study that showed high frequency of the ins/del allele in RIF group, thus the heterozygote genotype of ins/del leads to increase of RIF risk. ${ }^{21}$ Other study showed higher frequency of 14-bp insertion of the RIF patient group. ${ }^{10}$ However, differences between the current results and other studies could be due to different analysis method, different subgroups of RIF patients, different regional study, different sample size, and different time point of sample collection.

\section{Limitations}

Limitation of our study is the small size of patients group. In addition, the limited size of the kit was a hindrance to perform detection of polymorphism in both parents. It worth noting that the presence of soluble HLA-G in the embryo culture medium supernatant was proved by Desai et al. which suggests an association between implantation potential and embryonic secretion of sHLA-G. ${ }^{22}$ In the beginning of this study we investigated the presence of the mutation in two embryo culture media supernatants and the results were negative, eventually we replaced the supernatant samples with maternal blood samples to avoid many problems such as low sample size $(100 \mu \mathrm{l})$ which results a low DNA concentration, thus no conclusive results could be obtained. However, we recommend further studies of a larger scale to include both parents in this analysis of HLA-G polymorphisms, as well as the use of other samples including follicular fluid and culture supernatant samples.

\section{CONCLUSION}

We have indicated high frequency of the 14-bp ins/del 3'UTR HLA-G polymorphisms in both RIF patients and control groups, but no significant difference between the two groups was found. Our results suggest that there is no correlation between the polymorphism and implantation failure, thus it may be considered as a normal polymorphism and might not affect the levels of soluble HLA-G and there is no need to analyse the 14-bp ins/del 3'UTR HLA-G polymorphisms in middle-eastern women undergoing IVF.

\section{ACKNOWLEDGMENTS}

We appreciate the collaboration of the patients and express gratitude to Dr Adnan Alkhatib, Dr Hassan droubi, and Nawras Alhalabi for their kind collaboration. We also acknowledge the scientific research committee of Damascus university for providing a grant to carry out this study.

\section{Funding: No funding sources}

Conflict of interest: None declared

Ethical approval: The study was approved by the Institutional Ethics Committee

\section{REFERENCES}

1. Cao H, You D, Yuan M, Xi M. Hysteroscopy after repeated implantation failure of assisted reproductive technology: A meta-analysis. J Obstet Gynaecol Res. 2018;44(3):365-73.

2. Sharif K, Ghunaim S. Management of 273 cases of recurrent implantation failure: results of a combined evidence-based protocol. Reproduct Biomedic. 2010;21(3):373-80.

3. Potdar N, Gelbaya TA, Konje JC, Nardo LG. Adjunct low-molecular-weight heparin to improve live birth rate after recurrent implantation failure: a systematic review and meta-analysis. Human reproduction update. 2013;19(6):674-84.

4. Li Z, Wang X, Guan Y, Yu X, Liu J, Zhang Z. Uterine artery blood flow and microvessel density by vaginal color Doppler ultrasonography in embryo implantation failure. Experiment Therapeut Medic. 2017;14(5):4797-800.

5. Al-Turki HA. Hysteroscopy as an investigation tool in recurrent implantation failure in vitro fertilization. Sau Medic J. 2018;39(3):243. 
6. Boudjenah R, Molina-Gomes D, Wainer R, de Mazancourt P, Selva J, Vialard F. The vascular endothelial growth factor (VEGF)+ 405 G/C polymorphism and its relationship with recurrent implantation failure in women in an IVF programme with ICSI. J Assist Reproduct Genet. 2012;29(12):1415-20.

7. Lashley LEELO, Van Der Westerlaken L, Haasnoot G, Drabbels J, Spruyt-Gerritse M, Scherjon S, et al. Maternal HLA-C2 and 14 bp insertion in HLA-G is associated with recurrent implantation failure after in vitro fertilization treatment. Tissue Antige. 2014;84(6):536-44.

8. Fan W, Huang Z, Li S, Xiao Z. The HLA-G 14-bp polymorphism and recurrent implantation failure: a meta-analysis. J Assist Reproduct Genet. 2017;34(11):1559-65.

9. Vianna P, Dalmáz CA, Veit TD, Tedoldi C, Roisenberg I, Chies JAB. Immunogenetics of pregnancy: role of a 14-bp deletion in the maternal HLA-G gene in primiparous pre-eclamptic Brazilian women. Hum Immunol. 2007;68(8):668-74.

10. Aghdaie MH, Azarpira N, Kazemi K, Geramizadeh B, Darai M, Malekhoseini SA. Frequency of HLA-G exon 8 polymorphisms and kidney allograft outcome in Iranian population. Molecul Biol Report. 2011;38(5):3593-7.

11. Lynge Nilsson L, Djurisic S, Hviid TVF. Controlling the immunological crosstalk during conception and pregnancy: HLA-G in reproduction. Frontiers in immunology. 2014;5:198.

12. Niu Z, Wang L, Pang RT, Guo Y, Yeung WS, Yao Y. A meta-analysis of the impact of human leukocyte antigen-G on the outcomes of IVF/ICSI. Reproduct Bio Medic. 2017;34(6):611-8.

13. Nardi FdS, Slowik R, Wowk PF, da Silva JS, Gelmini GF, Michelon TF, et al. Analysis of HLA-G polymorphisms in couples with implantation failure. Am J Reproduct Immunol. 2012;68(6):507-14.

14. Mosaferi E, Majidi J, Mohammadian M, Babaloo Z, Monfaredan A, Baradaran B. HLA-G expression pattern: Reliable assessment for pregnancy outcome prediction. Advanc Pharmaceut Bullet. 2013;3(2):443.

15. Hviid TVF, Hylenius S, Rørbye C, Nielsen LG. HLA-G allelic variants are associated with differences in the HLA-G mRNA isoform profile and HLA-G mRNA levels. Immunogenet. 2003;55(2):63-79.

16. Hviid T, Hylenius S, Lindhard A, Christiansen O. Association between human leukocyte antigen-G genotype and success of in vitro fertilization and pregnancy outcome. Tissue Antige. 2004;64(1):66-9.

17. Kuroshli Z, Gourabi H, Bazrgar M, Sanati M, Esteki MZ. The Relationship between HLA-G Gene Polymorphisms and Repeated Implantation Failure in Infertile Couples Undergoing Assisted Reproductive Technique Repeated Implantation Failure in Infertile Couples Undergoing Assisted Reproductive Technique. Iran J Aller, Asthm Immunol. 2015;14(5):535-42.

18. Rizzo R, Fuzzi B, Stignani M, Criscuoli L, Melchiorri L, Dabizzi S, et al. Soluble HLA-G molecules in follicular fluid: a tool for oocyte selection in IVF? J Reproduct Immunol. 2007;74(12):133-42.

19. Ouji-Sageshima N, Yuui K, Nakanishi M, Takeda N, Odawara Y, Yamashita M, et al. sHLA-G and sHLAI levels in follicular fluid are not associated with successful implantation. J Reproduct Immunol. 2016;113:16-21.

20. Sipak-Szmigiel O, Cybulski C, Wokołorczyk D, Lubiński J, Kurzawa R, Bączkowski T, et al. HLA-G polymorphism and in vitro fertilization failure in a Polish population. Tissue Antig. 2009;73(4):348-52.

21. Enghelabifar M, Allafan S, Khayatzadeh J, Abadi KS, Nazarabadi MH, Moradi F, et al. Association of the maternal 14-bp insertion/deletion polymorphism in the histocompatibility leukocyte antigen $\mathrm{G}$ gene with recurrent implantation failure. Iran J Reproduct Medic. 2014;12(9):641.

22. Desai N, Filipovits J, Goldfarb J. Secretion of soluble HLA-G by day 3 human embryos associated with higher pregnancy and implantation rates: assay of culture media using a new ELISA kit. Reproduct Biomedic. 2006;13(2):272-7.

Cite this article as: Sulaiman R, Mohammad T, Alhalabi M. Analysis of 14-bp HLA-G polymorphism in middle-eastern women with recurrent implantation failure. Int J Reprod Contracept Obstet Gynecol 2020;9:4820-4. 